\title{
Cyclic adenosine monophosphate modulator supplementation on in vitro maturation of bovine oocytes
}

\author{
Minu Xaviour ${ }^{\star 1}$, R.S. Abhilash ${ }^{2}$, C. Jayakumar ${ }^{3}$, Amritha Aravind $^{4}$ and K. Raji ${ }^{5}$ \\ Department of Animal Reproduction, Gynaecology and Obstetrics, \\ College of Veterinary and Animal Sciences, Mannuthy, Thrissur- 680651 , \\ Kerala Veterinary and Animal Sciences University, Kerala, India.
}

Citation: Minu, X., Abhilash, R.S., Jayakumar, C., Amritha, A. and Raji, K. 2021. Cyclic adenosine monophosphate modulator supplementation on in vitro maturation of bovine oocytes. J. Vet. Anim. Sci. 52(4): 339-344ＤOI: https://doi.org/10.51966/jvas.2021.52.4.339-344

Received: 08.02.2021

Accepted: 03.04.2021

Published: 15.12 .2021

\begin{abstract}
Study evaluated the role of cAMP modulator (Forskolin and 3-isobutyl-1-methyl xanthine) supplementation on developmental competence of bovine oocytes. Cumulus oocyte complexes recovered from bovine ovaries of unknown reproductive status were used for the study. Oocytes retrieved by aspiration method were graded based on cumulus cell distribution and culture grade oocytes were selected for the study. A total of 414 culture grade oocytes were taken and divided into two groups. Group I constituted of 201 oocytes in which pre-maturation was carried out for a period of $2 \mathrm{~h}$. In group II, 213 oocytes were selected in which normal maturation was carried out. Maturation was assessed after $24 \mathrm{~h}$ of culture in $\mathrm{CO}_{2}$ incubator maintained at $38.5^{\circ} \mathrm{C}$ in 95 per cent humidified atmosphere of 5 per cent $\mathrm{CO}_{2}$. Fertilisation was carried out using frozen thawed semen and the presumptive zygotes were then transferred to culture media and cleavage was assessed $48 \mathrm{~h}$ after insemination. A significantly higher maturation rate $(p \leq 0.05)$ was observed in group I compared to group II $(86.85 \pm 1.19$ vs $79.88 \pm 2.67)$. There was a highly significant increase $(p$ $\leq 0.01)$ in cleavage rate in group I (65.92 \pm 1.23$)$ compared to group II (59.29 \pm 1.50$)$. A higher fertilisation rate was observed in group I (75.35 \pm 1.19$)$ than group II $(71.88 \pm 2.56)$. It could be concluded that pre-maturation with cAMP modulators improved the developmental competence of bovine oocytes.
\end{abstract}

Keywords: Oocyte, pre-maturation, cAMP modulators, forskolin

Running title: Pre- maturation of bovine oocytes with cAMP modulators

"Part of MVSc thesis submitted to Kerala Veterinary and Animal Sciences University, Pookode, Wayanad, Kerala

1. *MVSc Scholar and corresponding author: email- minuxaviour@gmail.com, Ph: 9400030458

2. Assistant Professor

3. Assistant Professor and Head (i/c)

4. Assistant Professor

5. Associate Professor, Department of Veterinary Physiology

Copyright: ( 2021 Minu et al. This is an open access article distributed under the terms of the Creative Commons Attribution 4.0 International License (http://creativecommons.org/licenses/by/4.0/), which permits unrestricted use, distribution, and reproduction in any medium, provided the original author and source are credited. 
During the last few decades, in vitro embryo production technology (IVEP) in bovines has received greater attention in animal husbandry. The procedure consists of three main steps starting from oocyte recovery, in vitro maturation and fertilisation of retrieved oocytes and in vitro culture of embryos. In vitro maturation is an assisted reproductive technology used to produce fully mature oocytes for wide range of applications like embryo production, human infertility treatments, transgenic technologies and cloning etc. It is a major strategic research tool in developmental and reproductive biology (Albuz et al., 2010). It involves gathering of immature oocytes from antral follicles and its subsequent culture under laboratory conditions to generate metaphase II oocytes.

Though IVEP is established, the efficiency is still limited to 30-40 per cent. Various researchers found that one main reason for this reduced efficiency is related to maturation of oocyte. Oocyte maturation involves both nuclear maturation as well as cytoplasmic maturation. Nuclear maturation is initiated from early foetal life onwards and it get arrested at diplotene stage of prophase I. Further maturation happens only after puberty up on LH surge. The oocytes will undergo synthesis and reorganization of cytoplasm during their selection and dominance phase (Luciano et al., 2018). Many factors play a role in holding oocytes in arrested stage, among that cyclic adenosine monophosphate is regarded as the major factor responsible for meiotic arrest. Though the major source of CAMP is from surrounding cumulus cells (CC), oocytes also synthesize some amount. There is a chain of exchange between CCs, gap junctions and oocyte. The cAMP produced from CCs is transferred to oocytes via gap junctions meaning gap junctional communication plays a major role in transport. Up on removal of oocytes from follicles, the gap junctional communication will be altered leading to drastic reduction in level of cAMP which will eventually culminate in activation of maturation promoting factor thereby resumption of meiosis (Pan and Li, 2019)

Oocytes exhibit a peculiar property of spontaneous resumption of meiosis when taken out of follicles. This occurs due to rapid drop in levels of cAMP. Oocytes retrieved for in vitro studies have not got enough time for synthesis and reorganization because they are taken out of antral follicles at different developmental stages. They won't be competent enough to hold future embryo. So an additional time apart from IVM is essential for cytoplasmic maturation. This can be made possible by incorporating cAMP modulators so that nuclear maturation can be stopped for a while. Forskolin and 3-isobutyl-1- methylxanthine (IBMX) are two cAMP modulators which act synergistically increasing intra oocyte cAMP levels. In view of above observations the present study was designed to investigate the effect of pre-maturation with forskolin and IBMX on developmental competence of oocytes.

\section{Materials and methods}

Bovine ovaries of unknown reproductive status were collected from slaughter house in antibiotic supplemented normal saline. Ovaries were washed, extra ovarian ligaments trimmed and after further washing maintained at $36-38^{\circ} \mathrm{C}$ till aspiration is completed. All visible surface follicles of $2-8 \mathrm{~mm}$ size were aspirated and collected in $10 \mathrm{~mL}$ test tube and kept undisturbed for 10min in incubator for settling. Later sediment in the bottom was pipetted out and COCs were identified under stereozoom microscope and graded as A, B, C and D.

\section{Experimental design}

A total of 414 culture quality oocytes of Grade A and B were selected for the study. Group I consisted of 201 oocytes kept for pre-maturation for a period of two hour before maturation and group II $(n=213)$ maturation was carried out by following standard protocol without pre-maturation with cAMP modulators. In group I, follicles were aspirated in to oocyte collection medium supplemented with $100 \mu \mathrm{M}$ forskolin and $500 \mu \mathrm{M}$ IBMX to prevent fall in cAMP assuming that resumption of meiosis starts up on removal of oocytes from follicles. Pre-maturation medium consisted of TCM199, supplemented with Earle's salts, foetal bovine serum (FBS), gentamicin, 500 $\mathrm{MM}$ IBMX and $100 \mu \mathrm{M}$ forskolin. After grading of oocytes, 
the same oocytes were transferred to prematuration droplets in a ratio of 10-20 oocytes per $100 \mu \mathrm{L}$ of maturation droplet. Oocytes were kept in pre-IVM medium for a period of $2 \mathrm{~h}$. After $2 \mathrm{~h}$, oocytes were washed serially in washing medium and transferred to maturation droplet. In group II, cumulus oocyte complexes were identified using stereozoom microscope and transferred to washing medium. After serial washings culture quality oocytes were transferred to maturation droplet (without prematuration) in a ratio of 10-20 oocytes per 100 $\mu \mathrm{L}$ of maturation droplet.

Maturation medium consist of TCM-199 (HEPES modified) supplemented with $\mathrm{FSH}$, estradiol-17 $\beta$, sodium pyruvate, L-glutamine, gentamicin sulphate and FBS. The culture condition set for the study was $38.5^{\circ} \mathrm{C}$ in 95 per cent humidified atmosphere of 5 per cent $\mathrm{CO}_{2}$. Maturation was assessed after $24 \mathrm{~h}$ of culture by cumulus cells expansion and first polar body extrusion. Fertilisation was carried out using frozen thawed semen. Sperm oocyte co-incubation was carried out for a period of $18 \mathrm{~h}$. The presumptive zygotes were then transferred to culture droplets after $18 \mathrm{~h}$ of incubation. Culture media consisted of SOF supplemented with BSA, Sodium pyruvate, essential and nonessential amino acids and gentamicin sulphate. Cleavage and fertilisation were evaluated $48 \mathrm{~h}$ post-insemination under inverted microscope at 40x magnification

\section{Results and discussion}

A total of 123 bovine slaughter ovaries of unknown reproductive status retrieved from slaughter house were subjected to follicular aspiration. A total of 554 visible surface follicles having 2- $8 \mathrm{~mm}$ diameter were aspirated. Mean number of follicles aspirated per ovary in the present study was $4.35 \pm 0.24$. The result was comparable with that of Rakshitha (2019) who obtained $5.58 \pm 0.23$ follicles per ovary and was lower than the observations of Manik et al. (2003) who observed $6.8 \pm 0.7$ follicles per ovary. The difference in the number of follicles found in ovaries can be due to the variations in the age of animal, breed, climatic conditions, presence or absence of corpus luteum in the ovary, nutritional, genetic and reproductive status of the animal (Singh et al., 2001)

The recovery rate of oocytes obtained in the present study was $87.88 \pm 1.50$ per cent. Oocyte recovery rate found in the study was is in accordance with Rakshitha (2019), who obtained recovery rate as 86.44 per cent. However, researchers like Singh et al. (2001) and Boonkong et al. (2012) obtained lower recovery rates of 67 and 58.6 per cent respectively. The variability in quality of oocytes obtained in the present study compared with other findings might be due to stage of the cycle at the time of slaughter, size and functional status of follicle, age, season, nutritional status and health condition of the animal (Nandi et al., 2002 and Sianturi et al., 2002).

There was a significantly higher maturation rate $(p \leq 0.05)$ was observed in group I (Fig. 1) compared to control group (86.85 \pm 1.19 vs $79.88 \pm 2.67$ ) (Table. 1 and Fig. 2 ) and a highly significant $(p \leq 0.01)$ increase $(p \leq 0.01)$ in cleavage rate was observed in group I (65.92 $\pm 1.24)$ compared to group II $(59.29 \pm 1.50)$ (Fig.3) The in vitro fertilisation rate of oocytes in experimental groups were $75.35 \pm 1.19$, and $71.88 \pm 2.57$ in group I, and II respectively. Even though there is no significant difference in fertilisation rate between experimental groups, a higher fertilisation rate was obtained in group I $(75.35 \pm 1.19 \%)$ than control $(71.88 \pm 2.57 \%)$.

Table. 1. Comparison of in vitro maturation rate in group I and II

\begin{tabular}{|c|c|c|c|c|c|}
\hline \multirow[b]{2}{*}{$\begin{array}{l}\text { Treatment } \\
\text { group }\end{array}$} & \multirow[b]{2}{*}{$\begin{array}{c}\text { No of oocytes } \\
\text { kept for } \\
\text { maturation }\end{array}$} & \multicolumn{2}{|c|}{ Maturation changes observed } & \multirow[b]{2}{*}{$\begin{array}{c}\text { Number of } \\
\text { matured } \\
\text { oocytes }\end{array}$} & \multirow[b]{2}{*}{$\begin{array}{c}\text { Maturation rate } \\
(\%)\end{array}$} \\
\hline & & $\begin{array}{c}\text { Cumulus cell } \\
\text { expansion } \\
(\%) \\
\end{array}$ & $\begin{array}{c}\text { First polar body } \\
\text { extrusion } \\
(\%)\end{array}$ & & \\
\hline Group I & 201 & $79.88 \pm 1.69(161)$ & $6.96 \pm 1.31(14)$ & 175 & $86.85 \pm 1.19^{a}$ \\
\hline Group II & 213 & $71.88 \pm 2.66(151)$ & $9.65 \pm 2.07(20)$ & 171 & $79.88 \pm 2.67^{b}$ \\
\hline
\end{tabular}

${ }^{\star *}$ Significant at 0.05 level. Means having different letter as superscript differ significantly 
The intra-oocyte cAMP concentration is regulated by the balance between the activity of two enzymes: adenylyl cyclase (AC) and phosphodiesterases (PDEs), responsible for CAMP synthesis and degradation, respectively. Cell to cell communications between follicular cells and the oocyte via gap junctions is critical for the completion of meiotic and cytoplasmic maturation (Gilchrist et al., 2007). It is via gap junctions that oocytes get essential nutrients, purines, nucleotides and metabolic support. Our results which indicate increased maturation, fertilisation and cleavage rate showed that the meiotic inhibitory effect was augmented when forskolin was supplemented with IBMX. For this reason, it is assumed that the prolongation of GJC between oocyte and cumulus cells after the treatment of IBMX and forskolin could be attributable to the accumulation of CAMP in cumulus cells and oocytes. The delayed loss of GJC in turn increased the concentration of intra-oocyte cAMP, which finally delayed the progression of GVBD (Luciano et al., 2004; Thomas et al., 2004).

In the present study, cumulus expansion was found to be greater in CAMP treated group than compared to control group. Also there is a significant difference noticed in IVM rate. It clearly depicts that when oocytes are meiotically arrested by introducing high level of cAMP modulators, the cytoplasmic maturation is getting improved. Oocytes can continue the reconstruction of cytoplasmic machineries by the time. For improving the yield and quality of developing embryos, in vitro cessation of oocyte meiosis is necessary. During this resting time, the oocyte will find the opportunity to continue transcription of mRNA, post-translational modifications of proteins, modification of organelles which are essential to sustain normal fertilization and further embryonic development.

It has been clearly understood previously that the chemical mediation by cAMP anologues have positive effect on oocyte maturation (Albuz et al., 2010). The increased rate of maturation in CAMP treated group clearly depicts the positive effect of supplementation of cAMP modulators from the initial period of oocyte collection. Richani

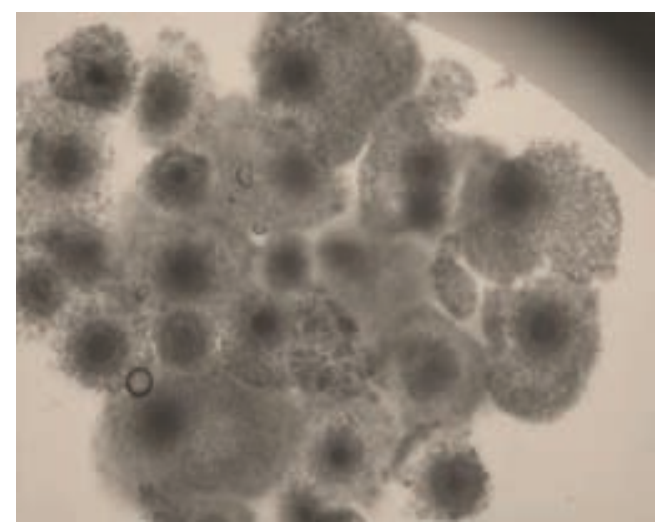

Fig. 1. Cumulus expansion noticed in group I

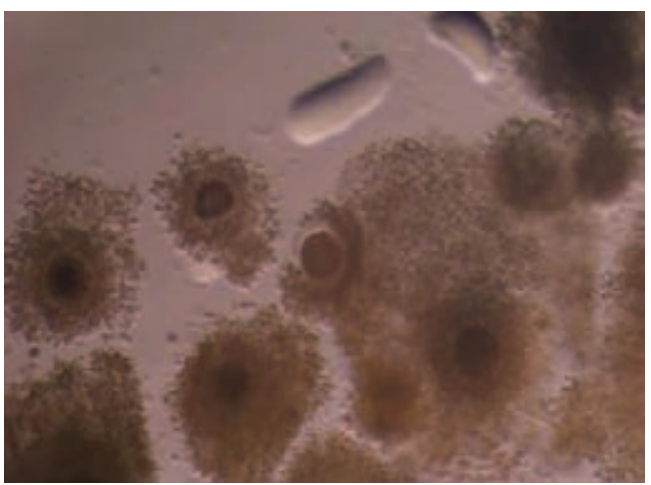

Fig. 2. Cumulus expansion noticed in group II

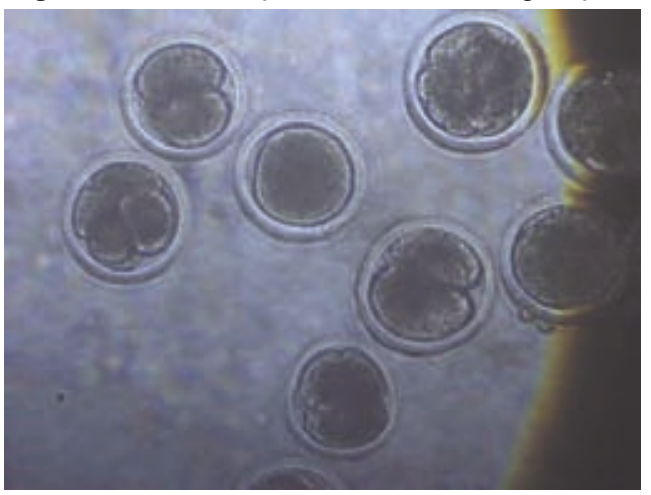

Fig. 3. Cleaved embryos

et al., (2014) found out that the blastocyst yield and quality of embryos was greater when the pre-IVM period was lengthened beyond $1 \mathrm{~h}$ suggesting that the duration of CAMPmodulated pre-IVM has important effects on oocyte developmental competence. Extending pre-IVM duration accelerated the time of 2-cell embryo development, which is indicated as a strong predictor of pregnancy success, the same was noticed by (Sugimura et al., 2012). 
Rose et al., (2013) carried out pre-maturation with forskolin and IBMX in ovine oocytes and came up with positive results showing greater developmental competence for pre-maturated oocytes.

\section{Conclusion}

Pre-maturation with cAMP modulators is a novel approach in the IVF field with a view to increase the output of IVM by improving cytoplasmic maturation and thereby increasing the developmental competence of oocytes. From the above findings, it is clear that supplementation of cAMP modulators in pre-IVM treatment has a positive effect on developmental competence of bovine oocytes. Hence, it can be concluded from this study that the efficiency of IVEP can be increased by incorporating pre-maturation before maturation so that cytoplasmic maturation can be improved.

\section{Acknowledgement}

The authors are thankful to the Dean, College of Veterinary and Animal Sciences, Mannuthy, Kerala Veterinary and Animal Sciences University for providing the facilities needed for carrying out this research work.

\section{Conflict of interest}

The authors declare that they have no conflict of interest.

\section{Reference}

Albuz, F.K., Sasseville, M., Lane, M., Armstrong, D. T., Thompson, J. G. and Gilchrist, R. B. 2010. Simulated physiological oocyte maturation (SPOM): a novel in vitro maturation system that substantially improves embryos yield and pregnancy outcomes. Hum. Reprod. 25: 29993011.

Boonkong, S., Navanukraw, C., Thammasiri, J., Jaikan, W. and Uriyapongson, S. 2012. Effect of culture media on fertilization rate in bovine and caprine embryos production. Int. J. Environ. Rural Dev. 2: 56-60.
Gilchrist, R. B., Zeng, H. T., Wang, X., Richani, D., Smitz, J. and Thompson, J. G. 2007. Re-evaluation and evolution of the simulated physiological oocyte maturation system. Theriogenology. 84: 656- 657.

Luciano, A., Franciosi, F., Barros, R., Dieci, C. and Lodde, V. 2018. The variable success of in vitro maturation: can we do better?. Anim. Reprod. 15: 727-736.

Luciano, A.M., Modina, S., Vassena, R., Milanesi, E., Lauria, A. and Gandolfi, F. 2004. Role of intracellular Cyclic Adenosine $\quad 3$ ',5'-monophosphate concentration and oocyte-cumulus cells communications on the acquisition of the developmental competence during in vitro maturation of bovine oocyte. Biol. Reprod. 70: 465 - 472.

Manik, R. S., Singla, S. K. and Palta, P. 2003. Collection of oocytes through trans vaginal ultrasound-guided aspiration of follicles in an Indian breed of cattle. Anim. Reprod. Sci. 76: 155-161.

Nandi, S., Ravindranatha, B. M., Gupta, P. S. P. and Sarma, P. V. 2002. Timing of sequential changes in cumulus cells and first polar body extrusion during in vitro maturation of buffalo oocytes. Theriogenology. 57: 1151-1159.

Pan, B. and Li, J. 2019. The art of oocyte meiotic arrest regulation. Reprod. Biol. Endocrinol. 17: 8.

Rakshitha, P.2019. Influence of dexamethasone and epidermal growth factor supplementation in synthetic oviductal fluid medium on in vitro bovine embryo development. M.V.Sc thesis, Kerala Veterinary and Animal Sciences University, Pookode, 88p.

Richani, D., Wang, X., Zeng, H. T., Smitz, J., Thompson, J. G. and Gilchrist, R. B. 2014. Pre-maturation with cAMP modulators in conjunction with EGFlike peptides during in vitro maturation enhances mouse oocyte developmental 
competence. Mol. Reprod. Dev. 81: 422435.

Rose, R. D., Gilchrist, R. B., Kelly, J. M., Thompson, J. G. and Sutton-McDowall, M. L. 2013. Regulation of sheep oocyte maturation using CAMP modulators. Theriogenology. 79: 142-148.

Sianturi, R.G., Thein, M., Wahid, H.andRosnina, Y. C. 2002. Effect of collection technique on yield of bovine oocytes and the development potential of oocytes from different grades of oocytes. Indonesian J. Anim. Vet. Sci. 7: 188-193.

Singh, S., Dhanda, O. P. and Malik, R. K. 2001. Effect of the presence of corpus luteum on ooctye recovery and subsequent in vitro maturation and fertilization in buffaloes. Asian Aust. J Anim. Sci. 12: 1675-1677.

Sugimura, S., Kobayashi, S., Hashiyada, Y., Ohtake, M., Kaneda, M. and Yamanouchi, T. 2012. Follicular growthstimulated cows provide favorable oocytes for producing cloned embryos. Cell Reprogram. 14: 29-37.

Thomas, R. E., Armstrong, D. T. and Gilchrist, R. B. 2004. Bovine cumulus cell-oocyte gap junctional communication during in vitro maturation in response to manipulation of cell-specific cyclic adenosine 3', 5'monophosphate levels. Biol. Reprod. 70: 548-556. 